\title{
A brief history of the DNA repair field
}

\author{
Errol C Friedberg ${ }^{1}$ \\ ${ }^{1}$ Laboratory of Molecular Pathology, Department of Pathology, University of Texas Southwestern Medical Center at Dallas, Dallas, \\ TX, USA
}
The history of the repair of damaged DNA can be traced to the mid-1930s. Since then multiple DNA repair mecha- nisms, as well as other biological responses to DNA damage, have been discovered and their regulation has been studied. This article briefly recounts the early history of this field.

Keywords: DNA repair, biological responses to DNA damage, ultraviolet light, excision repair, enzymatic photoreactivation, mismatch repair, DNA damage tolerance, recombination

Cell Research (2008) 18:3-7. doi: 10.1038/cr.2007.113; published online 24 December 2007

\section{Introduction}

As pointed out in numerous reviews of the topic of DNA repair [1], a full appreciation of the instability inherent in the chemistry of DNA and of its reactivity with a plethora of chemical and physical agents emerged surprisingly late after the elucidation of the DNA structure by Crick and Watson. This surprise is reinforced by the realization that studies on the mutagenic effects of agents such as ionizing radiation and ultraviolet (UV) radiation date back to the 1930s and 1940s [2].

\section{Early glimpses of biological responses to DNA dam- age}

The accelerated synthesis of physics, chemistry and biology in the United States in the 1930s did much to focus attention on the harmful effects of radiation on biological organisms. The subsequent emergence of atomic weapons in that country and the deployment of multiple national laboratories for atomic energy research generated an important emphasis on radiation biology. Alexander Hollander became a leading American figure in this field, spending much of his career as director of the Biology Division of the Oak Ridge National Laboratory. The Oak Ridge radiobiology program included several scientists who later made important contributions to the DNA repair field, notably Richard B (Dick) Setlow, Jane Setlow, William (Bill) Kimball and R C (Jack) von Borstel.

Correspondence: Errol C Friedberg

E-mail: errol.friedberg@utsouthwestern.edu
By the early 1940s it was becoming evident that agents that elicit mutational changes (such as ionizing and UV radiation) interact with and cause damage to the genetic material of cells. Additionally, hints began to emerge that living organisms can recover from the lethal effects of such damage [3]. These advances notwithstanding, "a combination of intellectual biases and to a lesser extent political influences, constrained the emergence of DNA repair as an area of investigative inquiry in parallel with other aspects of gene function. [2]" For one thing, the discovery that the master blueprint of life - the genetic material - was made of DNA still lay a good 13 years ahead. "Genes were still presumed to be made of proteins and to be intrinsically stable. There was no imperative to consider them at special risk to environmental or spontaneous damage, and hence in need of special biochemical perturbations. Mutations were considered to be rare events that were of enormous pragmatic value for genetic studies, but their mechanism of origin was not obviously experimentally tractable. Recovery after exposure to X-rays and UV light was an anecdotal phenomenon at best, and at worst the province of government "scientists" who were primarily intent on gleaning useful biological applications for the militaristic use of radiation, a task for which they were lavishly supported. Thus, the first direct experimental evidence for DNA repair did not emerge until just before the middle of the 20th century, and it was not until almost a decade later that the term DNA repair was confidently and unambiguously incorporated into the lexicon of molecular and cellular biology." [2]

Through common usage, the term DNA repair has come to embrace the entirety of the multiple and diverse ways in which living cells respond to genetic insults [1]. But strictly 
speaking, DNA repair is a biochemical term that defines biological processes during which alterations in the chemistry of DNA (DNA damage) are removed and the integrity of the genome is restored. The broader field is now more appropriately referred to as the field of biological responses to DNA damage, and in this context even the term "DNA damage" has been broadened to include phenomena such as the arrest of DNA synthesis in the absence of defined DNA damage.

\section{The discovery of DNA repair - enzymatic photore- activation}

Damage to DNA can be biochemically repaired by a number of mechanisms that are mechanistically distinct. Hence, many different types of true DNA repair exist [1]. Providentially, the first DNA repair mechanism to be discovered, enzymatic photoreactivation (EPR), is also believed to be the first DNA repair mechanism that evolved in nature. EPR is a process by which cyclobutane pyrimidine dimers in DNA, which are generated by exposure to UV radiation and which potently block both DNA replication and transcription, are removed from the genome. In this particular DNA repair mode, the removal of DNA damage results in the enzyme-catalyzed monomerization of pyrimidine dimers [1].

EPR was independently discovered in the late 1940s in two American laboratories. In both cases the discovery was incidental to the study of DNA repair and its emergence in the laboratory was initially a source of significant frustration to the investigators involved, both of whom had their experimental sights set elsewhere [2]. Albert Kelner was a young postdoctoral fellow at the Cold Spring Harbor Laboratory working with Miloslav Demerec. In the wake of the exciting discovery of penicillin, Kelner was attempting to generate mutants of the fungus Streptomyces griseus in the hope of identifying strains that produced novel and more effective antibiotics. Since UV light was then a fairly standard way of generating mutations in microorganisms, Kelner exposed both Escherichia coli and S. griseus cells on agar plates to various doses of UV radiation in order to calibrate his experimental system. To his considerable frustration, he obtained survival yields that were seriously irreproducible. By dogged persistence, Kelner [4] eventually identified exposure of his agar plates to fluorescent lights in the laboratory as the source of his variable results and went on to demonstrate that cells exposed to UV radiation underwent considerable rescue of viability if they were subsequently exposed to visible light. At the time when Kelner was trying to make sense of his baffling observations, the future Nobel laureate Renato Dulbecco was a postdoctoral fellow in Salvador Luria's laboratory at the University of Indiana in Bloomington, Indiana. (One of Dulbecco's laboratory colleagues was the young graduate student James (Jim) Watson, whose $\mathrm{PhD}$ thesis work demonstrated that photoreactivation was not observed when cells were exposed to ionizing radiation instead of UV radiation [2].) Luria, who together with Max Delbruck founded the famous "phage group" in the United States, was using UV radiation as an experimental probe of gene function during bacteriophage replication. Like Kelner, Dulbecco was exasperated to find that when he stored stacks of agar plates containing UV-irradiated phage-infected cells on his bench, the number of phage survivors varied enormously. Astutely, he noticed that plates on top of the stacks revealed the greatest number of phage plaques. He too eventually realized that he had discovered a light-dependent DNA repair mode [5], which was subsequently termed photoreactivation. It took several more years before photoreactivation was formally shown to be catalyzed by photoreactivating enzyme in a reaction that requires light in the visible range [6]. But importantly, the phenomenon of DNA repair was proved to exist.

\section{The discovery of excision repair}

As its name implies, excision repair is a process (actually a series of biochemically distinct processes) during which multiple types of DNA damage are excised from the genome, leaving gaps in the DNA duplex, which are themselves "repaired" by a non-semiconservative mode of DNA synthesis called repair synthesis [1]. In the late 1950s Ruth Hill, working at York University in Toronto, isolated a strain of $E$. coli that was abnormally especially sensitive to killing by UV light [7]. Additionally, several investigators reported that if cells treated with UV radiation were held in growth medium, or even buffer, before being plated, their survival was improved compared to cells plated immediately [1].

This effect, sometimes called liquid holding recovery, was independent of visible light, suggesting that some type of DNA repair other than EPR may transpire in bacteria. In the mid-1960s, Paul Howard-Flanders and his postdoctoral fellow Richard (Dick) Boyce at Yale University, and Richard (Dick) Setlow and his colleagues Paul Swenson and William (Bill) Carrier at the Oak Ridge National Laboratory, independently discovered that following exposure to UV light, bacteria such as $E$. coli remove small pieces of DNA from their genomes carrying UV radiation-induced lesions, such as pyrimidine dimers $[8,9]$. Howard-Flanders et al. [10] additionally isolated three mutant strains of $E$. coli (designated $u v r A, u v r B$ and $u v r C$ ) that were defective in this process. Since the mutations mapped to different regions of the $E$. coli genome, it was inferred that multiple gene products are involved in this excision process. At about the same time, Philip Hanawalt, a former graduate student with Setlow who was studying the effects of UV 
light on DNA replication with his graduate student David Pettijohn, reported that during the excision of pyrimidine dimers a form of non-semiconservative DNA synthesis occurs, which was eventually identified as the repair synthesis mode mentioned above [11]. Remarkably, considering the many difficulties of working with mammalian cells in culture in the mid-1960s, the phenomenon of excision repair of UV radiation-induced DNA damage was demonstrated in mammalian cells at about the same time as it was discovered in bacteria [12].

Over the next decade or so, studies in several laboratories demonstrated that most cells are endowed with multiple ways of excising base damage from the genome $[1,2]$. For example, believing that I was studying the same type of DNA repair mode just discussed, I discovered that the excision of pyrimidine dimers in E. coli cells infected with bacteriophage $\mathrm{T} 4$ (but not other T-phages) transpires by a different mechanism, one more or less simultaneously discovered by Mutsuo Sekigushi and his colleagues in Japan $[13,14]$. The same mechanism was independently discovered by the late Larry Grossman in the highly UV radiation-resistant bacterium $M$. luteus [15]. Grossman went on to show that this enzyme is in fact an unusual DNA glycosylase [16], a class of enzymes first discovered by Tomas Lindahl [17] and his colleagues during their search for the mechanism of the excision of uracil from DNA. Lindahl demonstrated that in contrast to pyrimidine dimers that are excised from the genome as small oligonucleotide fragments, uracil is excised as the free base. This led to the distinction between nucleotide excision repair (the process used for excising pyrimidine dimers and many other types of helix-distortive bulky adducts in DNA) and base excision repair (the process for excising uracil and other inappropriate bases in DNA, as well as many types of simple base damage, such as alkylated bases [1]). "As the decade of the 70s drew to a close, it became evident that nature exploits multiple mechanisms for the repair of base damage, sometimes for the repair of the same type of DNA damage. Particularly striking in this regard was the realization that the repair of pyrimidine dimers in DNA could be effected by three biochemically distinct pathways: photoreactivation, nucleotide excision repair and base excision repair initiated by the phage T4 or $M$. luteus pyrimidine dimer-DNA glycosylases. UV radiation damage from the Sun was clearly a potent selective agent during biological evolution." [1]

\section{Defective DNA repair can predispose to cancer}

The demonstration of non-semiconservative DNA synthesis in mammalian (including human) cells exposed to UV light [12] strongly suggested that this was repair synthesis operating during nucleotide excision repair. But to prove this definitively James (Jim) Cleaver at the University of California in San Francisco recognized the imperative of examining UV radiation-sensitive mammalian cell lines with the expectation that they would be defective in this repair synthesis mode. "I was working with [Bob] Painter shortly after he had discovered unscheduled DNA synthesis," recounted Cleaver [2]

We were trying to adapt Hanawalt's BrdU [bromodeoxyuridine] method to measure repair synthesis in mammalian cells. But the driving question was how to obtain UV-sensitive mammalian cell mutants to prove that the repair synthesis was really biologically related to excision repair. We were going to attempt to make such mutants ourselves. In April of 1967 I saw an article in the San Francisco Chronicle by the science writer David Perlman. It was a brief report of a clinical meeting - the 48th Annual Meeting of the American College of Physicians in San Francisco, and highlighted a talk on the genetics of human cancer by Henry Lynch. The article described xeroderma pigmentosum (XP) as a genetic disease with a predisposition to skin cancer and sensitivity to sunlight. I thought to myself. 'My word, here are God-given UV-sensitive mutants.' So we worked through the Dermatology Department at UCSF and got skin biopsies and cultures of XP patients. We got three cultures from three different patients with XP. Painter and I used the methods we had developed at that time for unscheduled DNA synthesis and Hanawalt's technique for repair replication. The results came up on each cell line right away $[1,18]$.

These results, independently confirmed by Dick Setlow and his colleagues [19], represented the first direct demonstration of the importance of maintaining the coding integrity of the human genome in preventing cells from undergoing neoplastic transformation. Indeed, the demonstration that XP individuals defective in nucleotide excision repair are highly predisposed to sunlight-induced skin cancer affords strong support of the somatic mutation theory of cancer. Further support of this theory and of the critical role of DNA repair in preventing cancer in humans came from the discovery and subsequent detailed investigation of yet another mode of excision repair, mismatch repair of DNA [1].

During normal semiconservative DNA synthesis, incorrect nucleotides are occasionally incorporated into the genome and constitute an important source of spontaneous mutations in cells unless the mismatched nucleotides are corrected. The repair of such lesions has a special requirement since cells must distinguish the newly synthesized DNA strand (containing the misincorporated nucleotide) from the parental (extant) strand (in which the mispaired nucleotide is the correct one). Matthew Meselson at Harvard University discovered that the mechanism for this DNA strand discrimination in bacteria such as E. coli involves methylation of adenine residues in GATC sequences in the genome [1, 2]. Paul Modrich and his colleagues at Duke University went on to demonstrate the enzymatic requirements for GATC methylation-dependent mismatch 
repair in bacteria [20] and subsequently in mammalian cells [1], and Modrich and others (including Richard Kolodner and Bert Vogelstein) were also instrumental in demonstrating that individuals who are genetically predisposed to a form of colon cancer called hereditary non-polyposis colon cancer are defective in mismatch repair [1]. Since then, several other human hereditary diseases have been shown to have their origins in defective DNA repair and/or other biological responses to DNA damage.

\section{The diversity of DNA repair mechanisms}

By the end of the 1970s, it was evident that cells have evolved multiple diverse mechanisms that center around two basic principles: the excision of base damage or its direct reversal. Earlier in this article, I discussed EPR as a primary example of the direct reversal of DNA damage. Other examples of this DNA repair mode have since been discovered, including the direct reversal of various types of alkylation damage $[1,2]$. In addition to nucleotide, base and mismatch repair, a form of excision repair called alternative excision repair was recently discovered [1] and Philip Hanawalt and his colleagues demonstrated that a specialized mode of nucleotide excision repair operates on the transcribed strand of transcriptionally active genes, the so-called transcription-coupled nucleotide excision repair $[1,2]$. In addition, numerous studies have demonstrated that both single- and double-strand breaks in DNA are subject to repair by a variety of mechanisms [1].

In general, it is fair to state that cells have evolved one or more mechanisms for repairing every type of naturally occurring DNA damage for which there has been sufficient time for natural selection. The study of the diversity of DNA damage associated with normal cellular metabolism and the exposure of cells to natural environmental agents such as UV radiation thus provides a cogent and frequently rewarding way of seeking new DNA repair pathways.

\section{The tolerance of DNA damage}

The detection and subsequent removal of DNA damage for the genomes of living cells are not completely efficient. Since damage is in general a random process, the DNA replication, transcription or recombination machineries may encounter sites of DNA damage before they are repaired. Such encounters may impede normal replication and transcription [1]. In order to cope with the deleterious effects of such encounters (such as cell death in the case of blocked DNA replication and sometimes in the face of blocked transcription) cells have evolved a series of biological responses referred to as DNA damage tolerance. As the term suggests, DNA damage tolerance allows cells to overcome the potentially lethal effects of blocked replica- tion and/or transcription until a time when the damage can be successfully removed.

The tolerance of DNA damage is frequently at the expense of mutations. Indeed, the various known modes of damage tolerance are potent contributors to the burden of spontaneous mutations that all replicating cells bear. An important historical contribution to our understanding of DNA damage tolerance was achieved in the mid-1970s when Miroslav Radman coordinated an extensive body of data from previous studies into a comprehensive hypothesis called the SOS hypothesis [1,2].

"Radman's paper enunciating the SOS hypothesis, entitled Phenomenology of an Inducible Mutagenic DNA Repair Pathway in E. coli: SOS Repair Hypothesis, was published in the proceedings of a meeting called "Molecular and Environmental Aspects of Mutagenesis" [2]. In it Radman systematically documented the (by then) mounting evidence for the existence of a number of DNA damageinducible phenotypes under control of the recA and lexA genes. He wrote

I would like to propose a general working hypothesis to account for phenomena and experiments briefly reviewed in the preceding paragraphs. Chronologically, this hypothesis preceded most of the reviewed experiments for which it has already served as the working hypothesis.

The principal idea is that $E$. coli possesses a DNA repair system which is repressed under normal physiological conditions but which can be induced by a variety of DNA lesions. Because of its 'response' to DNA-damaging treatments we call this hypothetical repair 'SOS repair'. The 'danger' signal which induces SOS repair is probably a temporary blockage of the normal DNA replication and possibly just the presence of DNA lesions in the cell. During the action of SOS repair mutation frequency is increased. The simplest assumption is that the SOS repair mechanism is error-prone; on the other hand, mutagenesis may be just a secondary consequence of physiological conditions under which SOS repair operates. In order for SOS repair to function it should require some specific genetic elements, the inducing signal and de novo protein synthesis. [2]

The late Harrison (Hatch) Echols at the University of California at Berkeley proposed a model to explain the SOS-regulated induction of mutations in E. coli cells exposed to UV radiation. The model suggested that when the DNA replication machinery is arrested at a site of unrepaired DNA damage (such as pyrimidine dimers), certain SOS-regulated genes encode proteins that interact with the stalled replication machinery in a manner that reduces their fidelity, thereby facilitating replication past the offending lesion. In the late 1980s and early 1990s, it was shown that the SOS-regulated genes identified in Echols's hypothesis are in fact specialized low-fidelity DNA polymerases that promote low-fidelity replication across the offending lesion, the so-called translesion DNA synthesis (TLS). Their markedly reduced fidelity allows the replicative bypass of 
sites of DNA damage, but with a high probability of incorporating incorrect nucleotides [1]. The past decade and a half has witnessed the discovery that eukaryotes, especially higher organisms, are endowed with multiple specialized DNA polymerases. The markedly reduced fidelity of these polymerases relative to those that are required to replicate the genome allows them to bypass many types of template strand base damage. However, the precise mechanism of TLS in eukaryotes is biochemically complex and remains to be fully elucidated.

In addition to their ability to bypass base damage of the type that can arrest DNA replication and transcription, some specialized DNA polymerases have been implicated in a natural mutagenic phenomenon, namely somatic hypermutation involved in the generation of high-affinity antibodies in the immune system [1].

\section{Regulation and coordination of DNA repair and DNA damage tolerance}

There may well be a small number of minor DNA repair and DNA damage tolerance mechanisms yet to be discovered. However, as we move forward in the 21st century, much of the cutting edge research in the field of biological responses to DNA damage is appropriately focused in trying to understand the regulation of numerous DNA repair and DNA damage tolerance mechanisms. How is base and other forms of DNA damage sensed in cells? And once sensed, how are signals transmitted from the sensing machinery to the various cellular pathways that determine the cellular response to the damage? What determines which of the multiple repair and tolerance mechanisms known are deployed first? How many DNA damage-related checkpoints exist in cells traversing the cell cycle and how are these activated when cycling cells sustain DNA damage of various types? How do cells make the decision to undergo extensive biological responses designed to rescue their viability, or to die by apoptosis? These are just a few of the many contemporary questions that now challenge the DNA repair field and keep it a vibrant and exciting field of scientific endeavor that is now inextricably linked with mainstream molecular and cellular biology.

\section{Acknowledgments}

Thanks to Nicole Kosarek for careful reading of the manuscript.

\section{References}

1 Friedberg EC, Walker GC, Siede W, Wood RD, Schultz RA, Ellenberger T. DNA Repair and Mutagenesis. Washington, DC:
ASM Press, 2005.

2 Friedberg EC. Correcting the Blueprint of Life. An Historical Accounting of the Discovery DNA Repairing Mechanisms. Cold Spring Harbor, NY: Cold Spring Harbor Laboratory Press, 1997.

3 Hollaender A, Curtis JT. Effect of sublethal doses of monochromatic ultraviolet radiation on bacteria in liquid suspension. Proc Soc Exp Biol Med 1935; 33:61-62.

4 Kelner A. Effect of visible light on the recovery of Streptomyces griseus conidia from ultraviolet irradiation injury. Proc Natl Acad Sci USA 1949; 35:73-79.

5 Dulbecco R. Reactivation of ultraviolet-inactivated bacteriophage with visible light. Nature 1949; 163:949-950.

6 Goodgal SH, Rupert CS, Herriot R. In: WD McElroy, B Glass, eds. The chemical basis of heredity. Baltimore, MD: Johns Hopkins Press, 1957.

7 Hill RA. Radiation-sensitive mutant of Escherichia coli. Bichim Biophys Acta 1958; 30:636-637.

8 Setlow RB, Carrier WL. The disappearance of thymine dimers from DNA: an error-correcting mechanism. Proc Natl Acad Sci USA 1964; 51:226-231.

9 Boyce RP, Howard-Flanders P. Release of ultraviolet light-induced thymine dimers from DNA in E. coli K1-12. Proc Natl Acad Sci USA 1964; 51:293-300.

10 Howard-Flanders P, Boyce RP, Simson E, Theriot L. A genetic locus in $E$. coli $\mathrm{K} 12$ that controls the reactivation of UV-photoproducts associated with thymine in DNA. Proc Natl Acad Sci USA 1962; 48:2109-2115.

11 Pettijohn D, Hanawalt P. Evidence for repair-replication of ultraviolet damaged DNA in bacteria. J Mol Biol 1964; 9:395-410.

12 Rasmussen RE, Painter RB. Evidence for repair of ultraviolet damaged deoxyribonucleic acid in cultured mammalian cells. Nature 1964; 203:1360-1362.

13 Friedberg EC, King JJ. Endonucleolytic cleavage of UV-irradiated DNA controlled by the $\mathrm{V}+$ gene in phage T4. Biochim Biophys Res Commun 1969; 37:646-651.

14 Yasuda S, Sekiguchi M. T4 endonuclease involved in repair of DNA. Proc Natl Acad Sci USA 1970; 67:1839-1845.

15 Kaplan JC, Kushner SR, Grossman L. Enzymatic repair of DNA, 1. Purification of two enzymes involved in the excision of thymine dimers from ultraviolet-irradiated DNA. Proc Natl Acad Sci USA 1969; 63:144-151.

16 Haseltine WA, Gordon LK, Lindan CP, Grafstrom N, Shaper 1, Grossman L. Cleavage of pyrimidine dimers in specific DNA sequences by a pyrimidine dimer DNA glycosylase of $M$. luteus. Nature 1980; 285:634-641.

17 Lindahl T. An $N$-glycosidase from Escherichia coli that releases free uracil from DNA containing deaminated cytosine residues. Proc Natl Acad Sci USA 1974; 71:3649-3653.

18 Cleaver JE. Defective repair replication of DNA in xeroderma pigmentosum. Nature 1968; 218:652-656.

19 Setlow RB, Regan JD, German J, Carrier WL. Evidence that xeroderma pigmentosum cells do not perform the first step in the repair of ultraviolet damage to their DNA. Proc Natl Acad Sci USA 1969; 64:1035-1041.

20 Lu AL, Clark S, Modrich P. Methyl-directed repair of DNA base pair mismatches in vitro. Proc Natl Acad Sci USA 1983; 80:4639-4643. 